\title{
A Platform and Clinical Model to Enable Medicare's Chronic Care Management Program
}

\author{
William R. Mills, MD, ${ }^{1}$ Dimitri Poltavski, PhD, ${ }^{2}$ Mark Douglas, MFin, ${ }^{1}$ Lisa Owens, RN, \\ Andrea King, LPN, Jamie Roosa, MA, LPN, ${ }^{1}$ Jacqueline Pridham, BA, \\ Daniel Dzina, BS, ${ }^{1}$ and David Weber, PA, RN, RRT, MBA ${ }^{1}$
}

\begin{abstract}
In 2015, the Centers for Medicare \& Medicaid Services (CMS) implemented a new benefit called chronic care management (CCM). A recent CMS-commissioned study of the program showed that CCM is effective in increasing advance care planning and decreasing overall costs. Despite positive effects on care planning, utilization, and cost, the CCM program remains underutilized. The authors sought to develop a platform to enable scale of the CCM program, and to report outcomes associated with its use. A technology and integrated clinical staff platform was built to enable a scalable, evidence-based implementation of the Medicare CCM program. The model created care management data elements that were used to flag clinical and utilization risks such as falls, mortality, hospitalization and polypharmacy. In 2018, CCM support was provided for 26,500 patients. Logistic regression analyses were used to identify risk factors associated with hospitalization. The cohort experienced 2679 hospitalizations (184 admissions per 1000 patient months per year). Among patients residing in non-nursing home settings, a higher Gagne mortality risk was associated with a 32 times greater chance of being hospitalized. Other positive predictors of hospitalization included being a nursing home resident and being ambulatory without assistance. Negative predictors of hospitalization included being flagged as having a high hospitalization risk, and scoring in the low-risk category for falls or polypharmacy. This CCM model is a scalable method of supporting care management for people with multiple chronic conditions, and can help identify risk factors for hospitalization.
\end{abstract}

Keywords: chronic care management, predictors of hospitalization, CCM, multiple chronic conditions

\section{Introduction}

A PPROXIMATELY TWO THIRDS of the Medicare fee-forservice population have $\geq 2$ chronic conditions. ${ }^{1}$ People with multiple chronic conditions often have needs and risks arise in-between their medical provider appointments but, historically, the Medicare fee-for-service population has not had an "in-between visit" care management benefit. In 2015, the Centers for Medicare \& Medicaid Services (CMS) implemented a new fee-for-service benefit, called chronic care management (CCM), that intends to support care management needs of Medicare beneficiaries with $\geq 2$ chronic conditions expected to last at least 12 months or until death. ${ }^{2}$

In 2017, CMS commissioned Mathematica Policy Research to study the first 18 months of the CCM program to assess the program's adoption, characteristics, and impact on utilization and cost. ${ }^{3}$ The study showed that, compared to a matched control group not receiving CCM services, the CCM cohort had significantly higher rates of advance care planning, more visits with their primary care physician, and used more home health care. The CCM group was hospitalized at lower rates than the non-CCM population, used emergency department services less often, and, after excluding patients who only received 1 month of CCM, cost Medicare $\$ 95$ less per month than patients not receiving CCM. When accounting for the cost of the CCM payment itself, the authors estimated that the CCM group saved CMS more than $\$ 38$ million. The results were striking, especially because CCM participating practices used many different approaches to operationalizing the program.

Although the CMS CCM service elements (Table 1) define the areas required to be documented for each CCM episode for billing purposes, to the research team's knowledge,

\footnotetext{
${ }^{1}$ Chronic Care Management, Inc., Solon, Ohio.

${ }^{2}$ University of North Dakota, Grand Forks, North Dakota.

(c) William R. Mills et al, 2019; Published by Mary Ann Liebert, Inc. This Open Access article is distributed under the terms of the Creative Commons Attribution Noncommercial License (http://creativecommons.org/licenses/by-nc/4.0/) which permits any noncommercial use, distribution, and reproduction in any medium, provided the original author(s) and the source are cited.
} 
Table 1. Centers for Medicare \& Medicaid Services Chronic Care Management Service Elements

Service element
Initiating Visit
Structured Recording of Patient
Information Using Certified
Electronic Health Record (EHR)
Technology
$24 / 7$ Access \& Continuity of Care

Comprehensive Care Management

Comprehensive Care Plan

Management of Care Transitions

Home- and Community-Based Care Coordination

Enhanced Communication Opportunities

Patient Consent

\author{
Medical Decision Making
}

Description

Initiation during an AWV, IPPE, or face-to-face E/M visit (Level 4 or 5 visit not required), for new patients or patients not seen within 1 year prior to the commencement of CCM services.

Structured recording of demographics, problems, medications, and medication allergies using certified EHR technology. A full list of problems, medications, and medication allergies in the EHR must inform the care plan, care coordination, and ongoing clinical care.

Provide 24/7 access to physicians or other qualified health care professionals or clinical staff including providing patients/caregivers with a means to make contact with health care professionals in the practice to address urgent needs regardless of the time of day or day of week.

Continuity of care with a designated member of the care team with whom the patient is able to schedule successive routine appointments.

Care management for chronic conditions including systematic assessment of the patient's medical, functional, and psychosocial needs; system-based approaches to ensure timely receipt of all recommended preventive care services; medication reconciliation with review of adherence and potential interactions; and oversight of patient self-management of medications.

Creation, revision, and/or monitoring (as per code descriptors) of an electronic person-centered care plan based on a physical, mental, cognitive, psychosocial, functional, an environmental (re)assessment and an inventory of resources and supports; a comprehensive care plan for all health issues with a particular focus on the chronic conditions being managed.

Must at least electronically capture care plan information, and make this information available timely within and outside the billing practice as appropriate. Share care plan information electronically (can include fax) and timely within and outside the billing practice to individuals involved in the patient's care. A copy of the plan of care must be given to the patient and/or caregiver.

Management of care transitions between and among health care providers and settings, including referrals to other clinicians; follow-up after an emergency department visit; and follow-up after discharges from hospitals, skilled nursing facilities, or other health care facilities

Create and exchange/transmit continuity of care document(s) timely with other practitioners and providers.

Coordination with home- and community-based clinical service providers.

Communication to and from home and community-based providers regarding the patient's psychosocial needs, and functional deficits must be documented in the patient's medical record.

Enhanced opportunities for the patient and any caregiver to communicate with the practitioner regarding the patient's care through not only telephone access, but also through the use of secure messaging, Internet, or other asynchronous nonface-to-face consultation methods.

Inform the patient of the availability of CCM services; that only one practitioner can furnish and be paid for these services during a calendar month, and of their right to stop the CCM services at any time (effective at the end of the calendar month).

Document in the patient's medical record that the required information was explained and whether the patient accepted or declined the services.

Complex CCM services require and include medical decision making of moderate to high complexity (by the physician or other billing practitioner).

AWV, annual wellness visit; CCM, chronic care management; E/M, evaluation and management; EHR, electronic health record; IPPE, initial preventive physical examination.

there have been no reports studying a cohort of patients' care managed by a single, evidence-based, CCM delivery model. Despite the positive effects on goal-directed care, utilization, and cost, and positive patient and provider feedback, the CCM program remains grossly underutilized. ${ }^{4-7}$

The primary aim of this study was to develop a technology and integrated clinical staff platform (Chronic Care Management, Inc, Cleveland, $\mathrm{OH}[\mathrm{CCMI}]$ ) to enable a scalable, evidence-based implementation of the Medicare CCM program and to examine factors associated with hospitalization.

\section{Methods \\ Incorporation of evidence-based assessments into the technology platform to support the CCM workflow}

In order to support the systematic use of evidence-based care management within the CCM program, the research team performed a review of care management studies, guidelines, and protocols that support an "in-between visit" care management approach. The team selected candidate 
assessments and workflow modules for integration into the new technology platform based on $(a)$ evidence of their utility in Medicare-age populations and $(b)$ whether it could support the non-face-to-face nature of the CMS CCM program. Criteria that also influenced selection included: (c) whether or not an assessment tool could provide discrete data, $(d)$ whether the results of a given assessment could trigger identification of risk, and $(e)$ whether the results could trigger an action that could serve to support a patient in a way that could better align goals of care, increase quality, or lower avoidable acute care utilization.

Fourteen core care management modules were built into the core CCM technology-supported workflow (Figure 1a). Available evidence was used to support assessments of potential patient risks such as: mortality, ${ }^{8,9}$ hospitalization, ${ }^{10}$ falls, ${ }^{11}$ depression, ${ }^{12,13}$ goals of care,${ }^{14}$ functional status, ${ }^{10}$ medication management, ${ }^{10}$ polypharmacy, ${ }^{15}$ and unmet social needs. ${ }^{16}$

\section{Model of supporting medical groups}

Medical groups contracted with CCMI for use of CCMI's technology and clinical staff to support their patients' CCM needs. The medical group identified patients meeting CCM inclusion criteria, obtained consent, and notified CCMI of each patient's enrollment. Medical group practitioners continued to see enrolled patients for regular medical visits in their chosen care settings (ambulatory clinic, home visit, longterm care nursing home visit) throughout the study period.

\section{Clinical staff model}

Clinical staff members supported practitioners and their patients with non-face-to-face support, under the CCM program's incident-to, general supervision requirements. The CCMI workforce included licensed nurses and medical assistants as primary chronic care coordinators. Each fulltime chronic care coordinator was assigned an average of 400 patients. Chronic care coordinators were grouped into teams of between 6 and 8 , and each team was supervised by a licensed nurse who was experienced in CCM. The nurse team leader provided education, scheduling, and quality review for the coordinators, and served as the main communicator between the team and the medical practice.

\section{Data sources}

To complete a CCM episode, chronic care coordinators used several data sources in order to complete the assessments. Access to the medical group's electronic health record allowed capture of diagnoses, medications, visit notes, medical histories, and other clinical data. In settings where CCM patients had nursing care (assisted living, long-term care nursing), nursing notes also were incorporated into

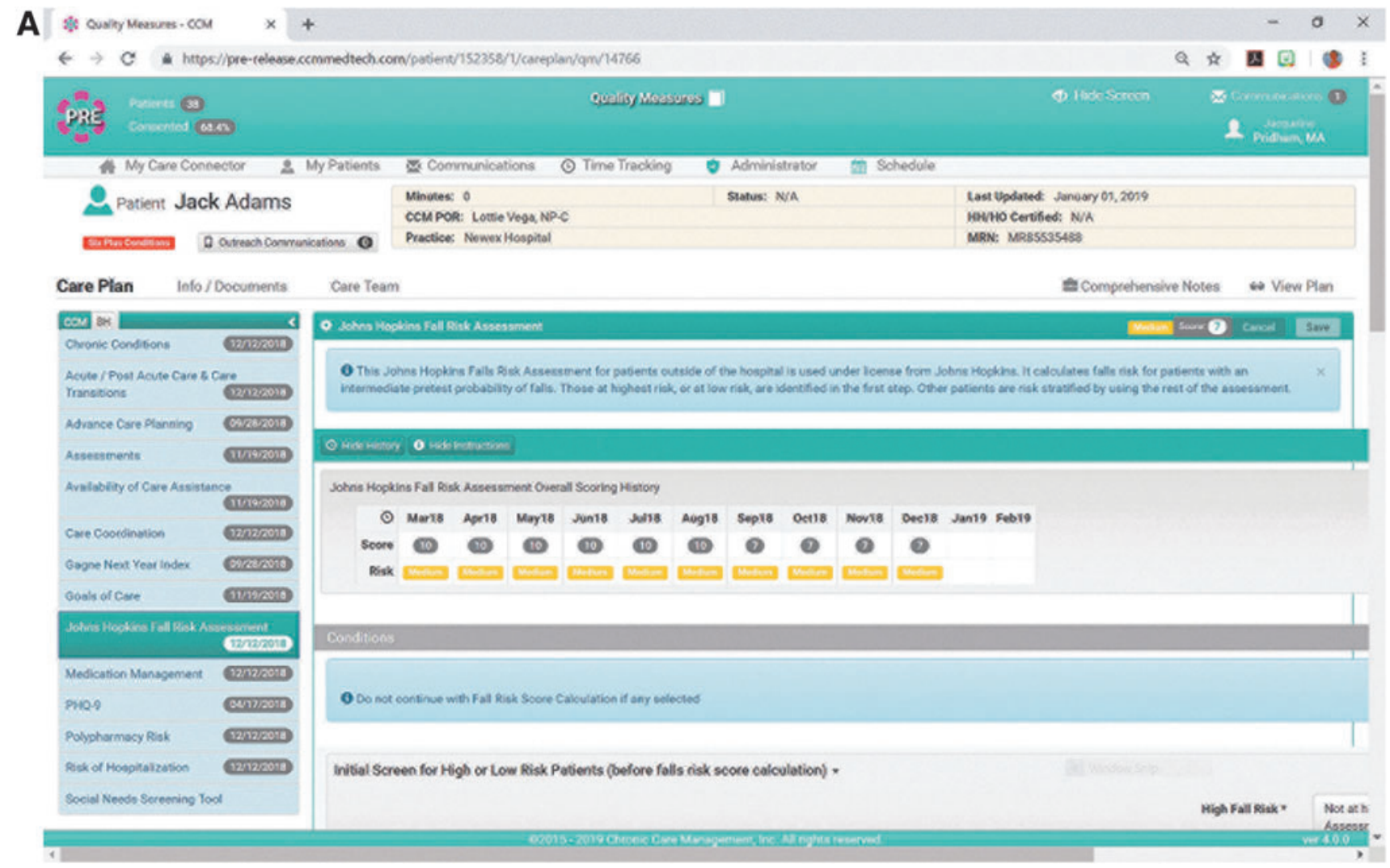

FIG. 1. Depiction of the Chronic Care Management, Inc. technology platform used to support evidence-based care management workflow and patient engagement.

(A) Assessments integrated into a technology platform supporting "in-between visit" chronic care management. 

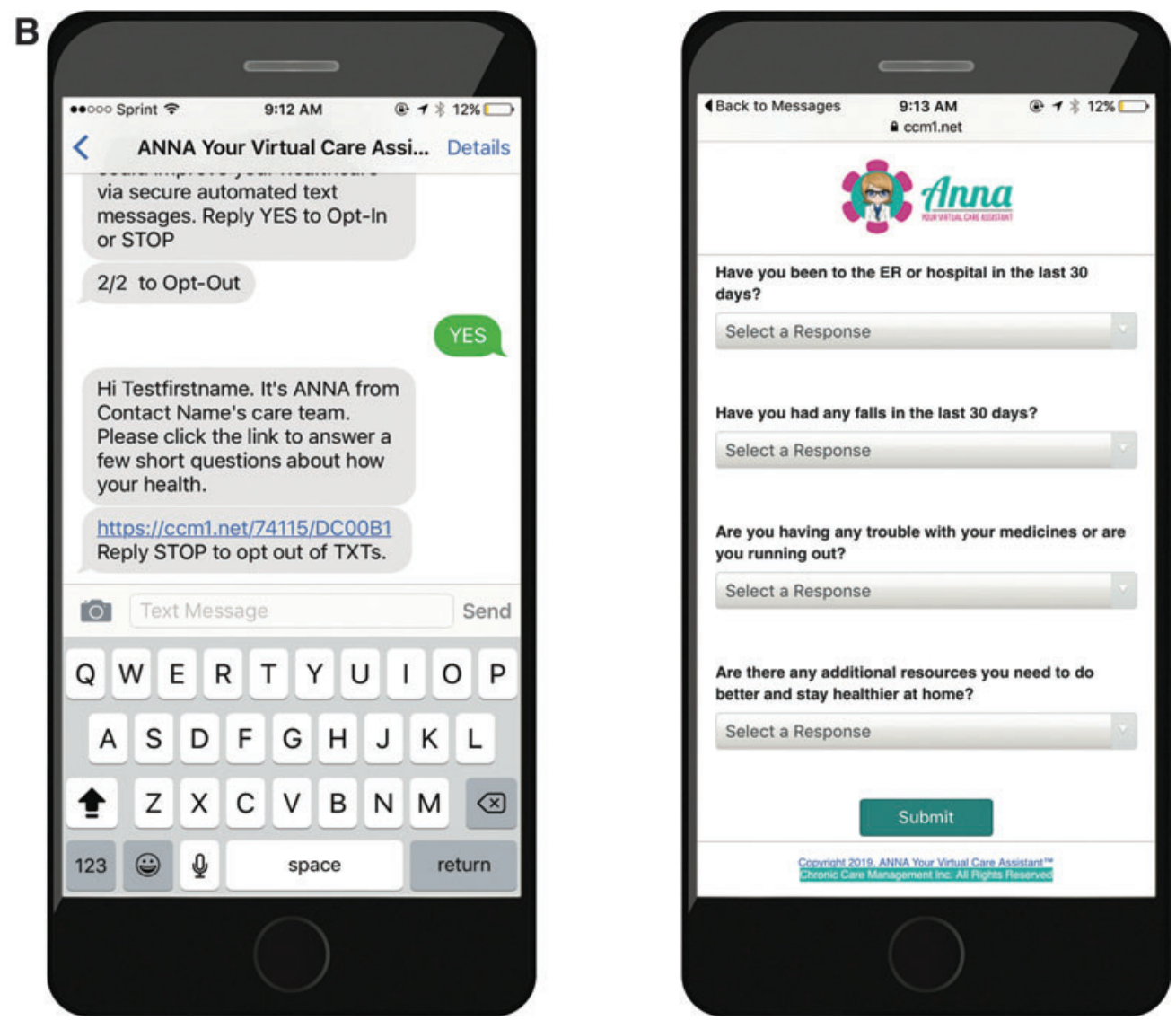

FIG. 1. (B) Patient engagement methodology, showing ANNA Your Virtual Care Assistant.

CCM data review. When possible, patient engagement data (to be described further) also were included as a data source used to develop the CCM episode.

\section{Patient engagement}

A monthly outreach was made to patients able to engage in care management outreach. Where a valid, short-message service (SMS)-capable number was provided, outreach was initiated with ANNA Your Virtual Care Assistant. ANNA is a technology developed by CCMI to deliver secure content and questionnaires to patients, initiated by a text message sent to their phone (Figure 1b). Patients who did not have a valid SMS-capable number but who could engage in CCM outreach were contacted by coordinators via telephone. Coordinators met with the nurse or caregiver of patients who were unable to engage with outreach attempts directly because of physical, mental, or cognitive limitation. The core care management questions shown in Figure 1b were utilized across outreach modalities.

\section{Practitioner engagement}

Practitioners were engaged in an initial CCM implementation meeting during which CCM data handling, triage, and communication preferences were formalized. When a CCM episode resulted in an identified risk, that data output was communicated to the medical practice based on the triage protocol. Practitioners were able to review their patients' CCM episode care plans, and could refer patients to resources that could help address an identified risk (Figure 2).

\section{Statistical analyses}

A binary logistic regression module of SPSS 24.0 statistical software (IBM Corp., Armonk, NY, 2016) was used in the CCM sample to identify the most robust predictors of hospitalization. Three groups of predictors were used: (1) age, sex, ambulatory status, and whether the patient lived in a long-term care nursing home; (2) presence of an advance care plan, number of CCM episodes; and (3) risk of hospitalization, number of chronic conditions expected to last for at least 12 months or until death, polypharmacy risk, Johns Hopkins Falls Risk Assessment (JHFRA) tool, and mortality risk within a year. Mortality risk was based on the Gagne mortality index for community-dwelling patients and Flacker mortality index for nursing home residents.

Logistic regression was chosen over multiple regression as the number of hospitalizations $>2$ (range 1-6) in a year accounted for only $4 \%$ of all hospitalizations $(n=2679)$ with 1 hospitalization comprising $82.6 \%$ of all hospitalizations. ${ }^{17-20}$ A backward elimination procedure was used, based on likelihood ratio test for estimation of individual parameters. The CCM sample resulted in the total of 25,032 non-missing data entries for the number of hospitalizations between January 1 and December 31 of 2018. A total of 2679 patients 


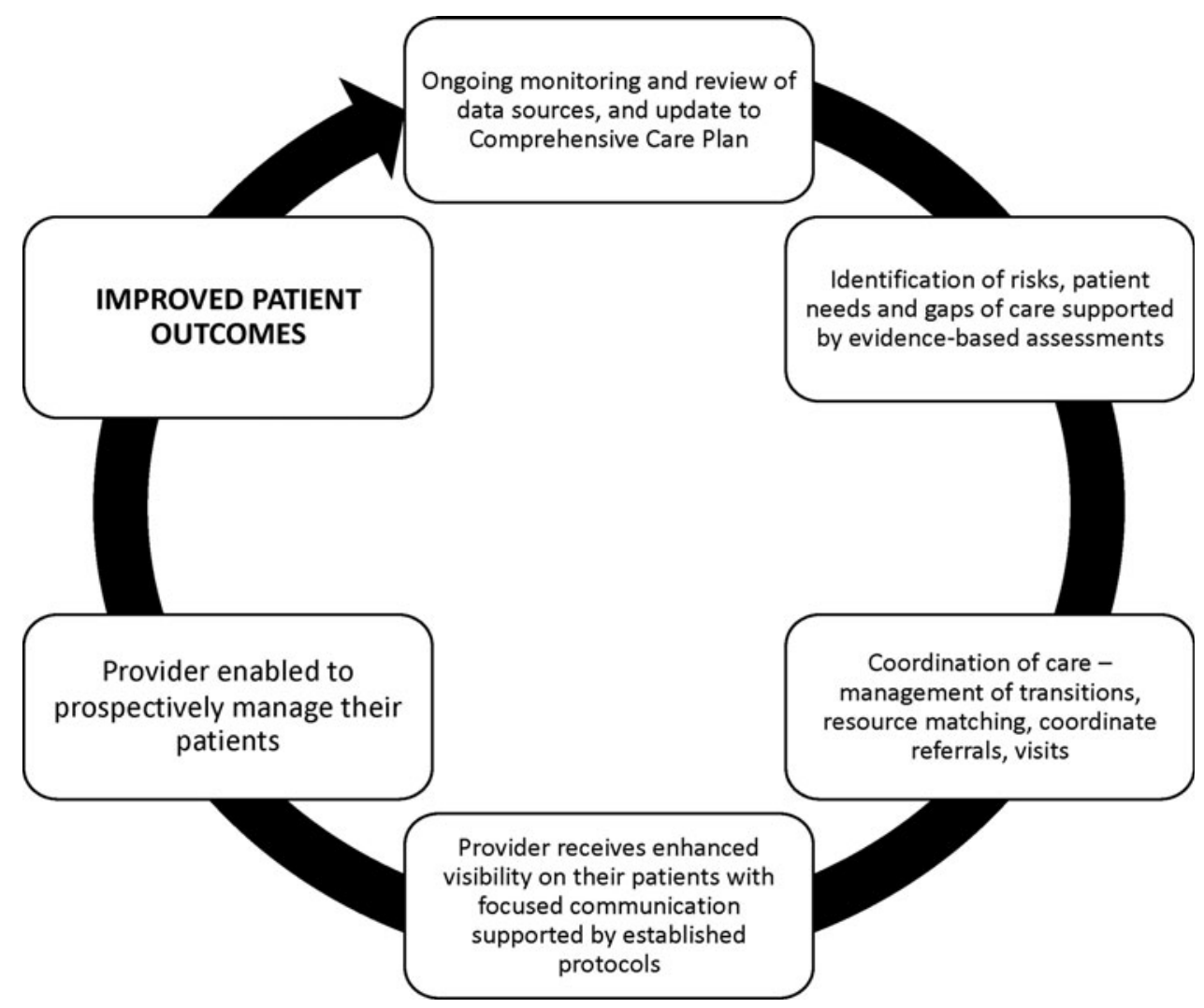

FIG. 2. Practice engagement methodology.

had at least 1 hospitalization in that time period (10.7\% of the sample).

The research team split the sample of nonhospitalized CCM patients in 2 equal halves. The team randomly sampled 2679 patients from one half, combined them with the sample of 2679 hospitalized patients, and developed a logistic regression model on this training sample. The team then saved the estimated parameters for the model and applied them to the testing sample, which contained 2679 patients randomly sampled from the other half of the nonhospitalized CCM patient pool. Application of the training model's parameters to the new sample resulted in estimated posterior probabilities of group membership in the testing sample. The team used these probabilities to classify participants and assessed the shrinkage of the testing model compared to the training model. ${ }^{21}$

Prior to saving logistic regression model parameters for the training sample, the research team removed all multivariate outliers that were identified based on standardized residual values exceeding 1.96, which is significant at $\alpha$ 0.05 . The team followed up the logistic regression analyses with receiver operating characteristic (ROC) curves on the most influential predictors in the model to assess how well individual predictors were able to discriminate between the 2 categories of dependent variable.

\section{Results}

Between January 1 and December 31, 2018, CCMI supported 26,500 patients in the described model (Table 2). The majority of patients resided in community settings, while less than 5\% lived in nursing homes in long-term care. The average age of CCM patients was $75(\mathrm{SD}=13)$; more than $60 \%$ were female. Most patients had $\geq 6$ chronic conditions expected to last 12 months or until death (Table 2). A primary circulatory chronic condition was present in $88 \%$ of the CCM sample $(n=23,335)$, followed by the presence of an endocrine/metabolic condition $(\mathrm{n}=12,162,46 \%)$ and/or gastroenterological condition $(n=11,687,44 \%)$. In total, 174,612 CCM episodes (CCM patient months) were performed on this cohort $(\mathrm{M}=6.6$ per patient, $\mathrm{SD}=3.9)$.

Risk assessments were performed using available clinical data during CCM episodes. More than $70 \%$ of patients were ambulatory without assistance (72\%), whereas less than $30 \%$ either needed assistance with ambulation or were nonambulatory (Table 2). More than half of the CCM population had an advance care plan on file. During 2018, 15\% of patients were flagged as high risk for hospitalization using the calculator adopted from the OASIS (Outcome and Assessment Information Set)-C2 question set. ${ }^{10}$ Approximately $40 \%$ of patients were found to be in the high risk for falls tier by using the JHFRA tool. ${ }^{11}$ Nearly $80 \%$ of patients were in the high-risk polypharmacy category ( $\geq 7$ medications) (Table 2). There were 2679 hospital admissions during 2018, corresponding to an overall hospitalization rate of 184 per 1000 patient months per year.

\section{Logistic regression analyses}

Preliminary diagnostics identified 1006 cases with missing values for at least 1 of the predictor variables. Additionally, 3 cases were identified as multivariate outliers 
Table 2. Characteristics of Patients Receiving Chronic Care Management Services Using Study PlatForm Between January 1, 2018, AND DECEMBER 31, 2018

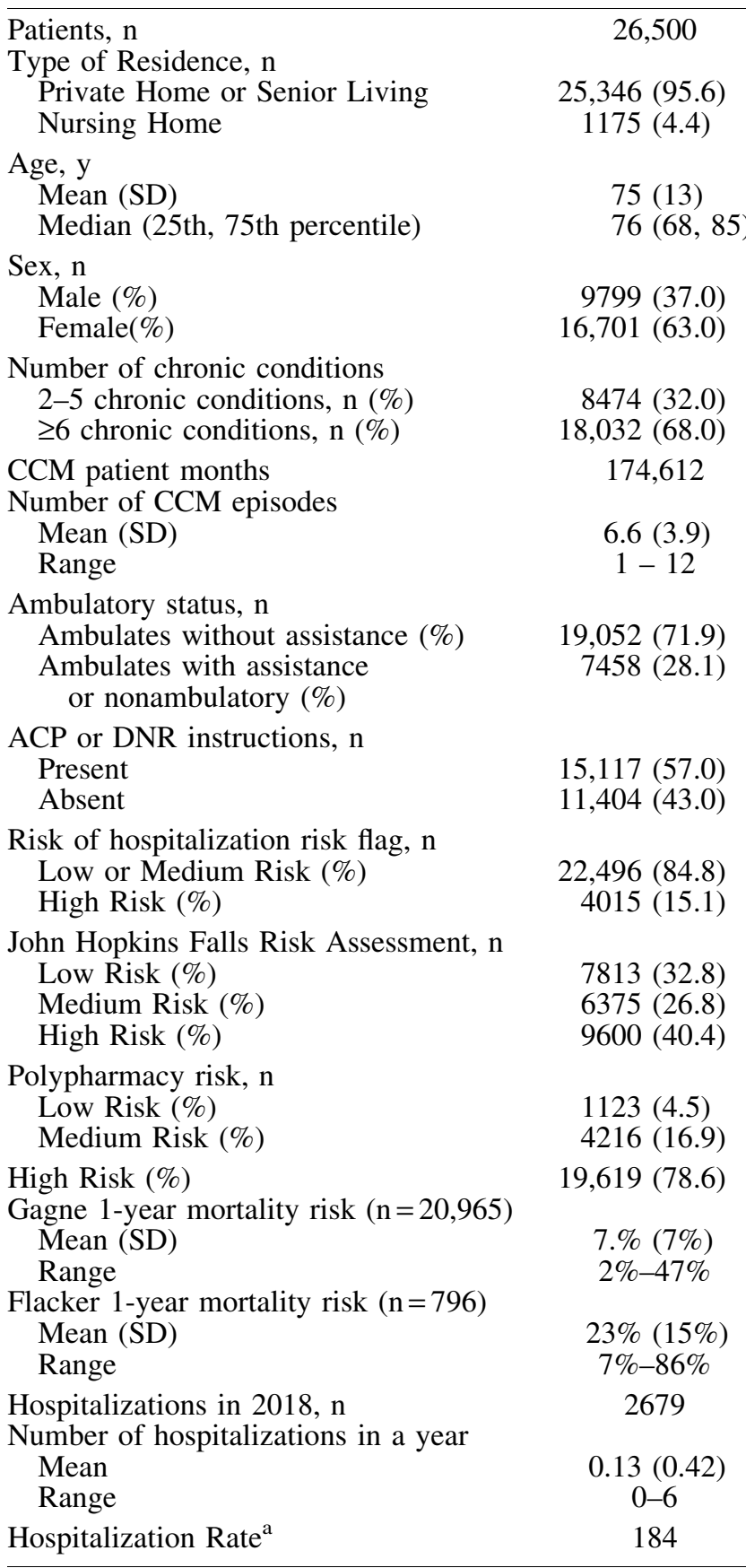

${ }^{\mathrm{a}}$ Hospitalization rate is expressed as number of hospital admissions per $1000 \mathrm{CCM}$ patient months per year.

ACP, advance care plan; CCM, chronic care management; DNR, do not resuscitate; SD, standard deviation.

and removed from the data set. The resultant training sample included 4352 patient records (2063 patients hospitalized at least once in 2018 and 2289 randomly selected from the nonhospitalized sample). The default null model thus had a proportional by chance accuracy rate of $50.1 \%$ in classifying patients as hospitalized.
Based on the stepwise backward elimination procedure, 7 of the 10 original predictors were retained in the final model. The fit of the model did not change significantly after removal of sex (step 1), presence of an advance care plan (Step 2), and number of chronic conditions expected to last for at least 12 months or until death. The remaining 7 predictors accounted for about $11 \%$ of the variability in hospitalization data (Nagelkerke $\mathrm{R}^{2}=0.113$ ), which was a statistically significant improvement in classification accuracy compared to the null model $\left(\chi^{2}=386.07 ; P<0.01\right)$. The final model had an overall classification accuracy of $62.3 \%$, which marginally satisfied the $25 \%$ criterion. The model did somewhat better when classifying individuals who were hospitalized (sensitivity $=67.4 \%$ ) than when classifying those who were not (specificity $=56.7 \%$ ).

Greater likelihood of hospitalization was associated with younger patient age, independent ambulation status, nursing home residence, a greater number of CCM episodes, and a greater 12-month predicted mortality risk. On the other hand, a lower likelihood of hospitalization was linked to low polypharmacy risk and low risk of falling as well as (counterintuitively) to an identified high risk of hospitalization. By far the greatest contributor to the model was the 12-month mortality risk. Patients with higher risk scores on this index were 17.15 times more likely to be hospitalized. Nursing home residents were 2.2 times more likely to be hospitalized compared to community-dwelling CCM patients. Independent ambulatory status increased chances of hospitalization by about $25 \%$, while low risk of falling decreased this likelihood by approximately $27 \%$. Correspondingly, those in the low-to-moderate polypharmacy risk categories were about $32 \%$ less likely to be hospitalized compared to the high-risk group. For specific odds ratios refer to Supplementary Table S1.

The research team followed up the logistic regression analyses with ROC curves for the most important predictor in the model (ie, 12-month mortality index) (Figure 3). On its own, although the predictor was statistically significant $(P<0.01)$, it showed modest utility in discriminating between those CCM patients who were hospitalized in 2018 versus those who were not (AUROC [area under the ROC] $=0.59 ; P<0.01)$.

\section{Additional logistic regression analyses}

Because type of residence and mortality index were the 2 most important predictors of hospitalization, the research team conducted follow-up logistic regression analyses within the patient population of each residence type (ie, community-dwelling or nursing home patients) using their respective mortality indexes (Gagne and Flacker) as separate predictors. Results showed that within the population of community-dwelling CCM patients included in the analysis ( $\mathrm{n}=4159 ; 2204$ hospitalized), the final model was significantly improved compared to the null $\left(\chi^{2}=347.28 ; P<0.01\right)$ and accounted for approximately $11 \%$ of the variance in the dependent variable (Nagelkerke $\mathrm{R}^{2}=0.11$ ). The proportional by chance increase in classification accuracy was $23.4 \%$ (from $50 \%$ in the null model to $61.7 \%$ in the final model). The model was very similar to the original combined sample model, with the Gagne mortality index being the most important predictor of hospitalization. Higher Gagne scores 


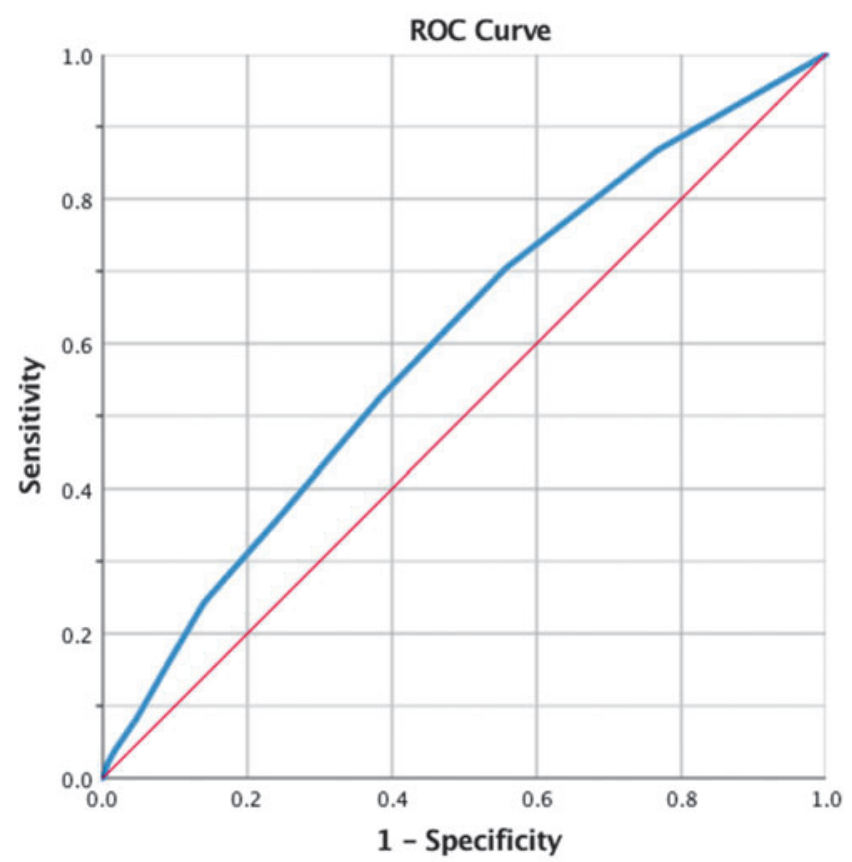

Diagonal segments are produced by ties.

FIG. 3. ROC curve for Gagne mortality risk index predicting hospitalization in community-dwelling CCM patients. CCM, chronic care management; ROC, receiver operating characteristic.

increased the risk of hospitalization 32.33 times. The ROC curve showed modest predictive utility for this risk factor with the AUCROC $=0.60$ and best cutoff scores between 0.04 and 0.06 (Youden's $\mathrm{J}=0.14$ ).

\section{Discussion}

The current study reports use of a standardized CCM workflow, enabled by a technology platform, and outcomes associated with its use on a cohort of 26,500 patients. The cohort studied was a multimorbid group, two thirds of whom had $\geq 6$ chronic conditions - a group noted to be among the costliest group of Medicare beneficiaries, ${ }^{22}$ costing a per capita annual average of more than $\$ 32,000$. Nearly $30 \%$ of CCM patients required assistance with ambulation, another high-risk, high-cost group. ${ }^{23}$ The group had, on average, 6.6 CCM episodes during 2018, a number similar to recent reports on $\mathrm{CCM}^{3}$ More than half of the cohort had an advance care plan $(57 \%)$. Comparatively, the CCM population in Shurrer's report ${ }^{3}$ was reported to have a $10 \%$ advance care planning rate, a number well above the Medicare population average of only $1 \%$.

These results show that by incorporating evidence-based risk or gap assessments into CCM workflow, typical gaps (eg, not having ACP on file) and risks (eg, falls risk) can be identified and addressed. The research team chose workflow tools that could be incorporated into the CCM workflow by clinical staff, with an aim of selecting assessments that could provide actionable, discrete, risk-stratified data output. Using the OASIS risk of hospitalization assessment tool, ${ }^{10}$ it was found that $15 \%$ of patients were flagged to be at high risk of hospitalization during 2018. For this high-risk group, the clinical staff team notified the client practice's physician or his/her designee. The research team recommended that the provider schedule a visit or perform non-visit-based care management outreach to high-risk patients to mitigate risk, but did not have uniform visibility into the actual process each client practice used. The team believes that proactive identification of hospitalization risk and communication of the risk to the practice may have led to this group being hospitalized at approximately half the rate of patients not in this high-risk tier.

A similar approach was taken when CCM patients were flagged as high risk for falls by the CCMI clinical staff using the JHFRA tool, where $40 \%$ of the CCM population was high risk. JHFRA was especially useful because of the visibility of its score components, which provide clinical targets for practitioners to decrease falls risk. ${ }^{11}$ For example, if a patient received 5 points for being on high risk for falls medications, the practitioner could deprescribe such meds, thereby decreasing the JHFRA predicted falls risk. Polypharmacy risk was captured simply as the total number of medications a CCM patient was prescribed, and patients on $\geq 7$ medications, shown to be at an $82 \%$ risk of an adverse medication interaction, ${ }^{15}$ were flagged as high risk. Twelvemonth predicted mortality rate was calculated using the Gagne index for patients living in the community, ${ }^{8}$ and using the Flacker index for patients living in a long-term care nursing home setting. ${ }^{9}$ Although this study was not structured to compare hospitalization rates experienced by CCM patients versus a non-CCM control group as Shurrer did, the present study CCM cohort did experierience low hospitalization rates (184 admits per $1000 \mathrm{CCM}$ patient months per year).

Overall, the logistic regression model was robust and moderately useful in identifying CCM patients who are likely to be hospitalized. The model was about $62.3 \%$ accurate in patient classification and had a sensitivity of $67.4 \%$. The $12-$ month mortality index based on the Gagne and Flacker scores for the respective populations was the biggest contributor to the model, followed by residence status, risk of falling, and polypharmacy risk. Interestingly, patients identified at high risk for hospitalization actually were $49 \%$ less likely to be hospitalized. In addition to the process of communicating hospitalization risk to client practices mentioned, the followup analyses also demonstrated that those identified at high risk of hospitalization had significantly $(t=-6.87, P<0.01)$ more $\mathrm{CCM}$ episodes $(\mathrm{M}=8.1, \mathrm{SD}=3.6)$ than those who were not listed as "high risk" ( $\mathrm{M}=7.2 ; \mathrm{SD}=3.7)$, which also may have mitigated the group's hospitalization rate.

Follow-up logistic regression analyses within each residence type showed that the greatest predictive power of the mortality index was driven primarily by the Gagne mortality risk score in the community-dwelling CCM patients, as the odds ratio of hospitalization increased in this sample from 17 to 32 when this indicator was analyzed separately. The Flacker mortality index was not a significant predictor of hospitalization among nursing home residents. Future studies are needed to further define other strong predictors of hospitalization. Providers should use predictors of hospitalization such as the Gagne mortality index, as well as others, in the context of their clinical judgment and experience.

In conclusion, this model of supporting CCM for Medicare-age patients is a scalable method of identifying gaps and risks that people with multiple chronic conditions commonly experience, and it was effective in identifying 
factors associated with hospitalization. Most notably, practices caring for Medicare-age patients with multiple chronic conditions who do not reside in nursing home settings should consider using the Gagne mortality index to stratify hospitalization risk. Finally, whether or not patients are in the Medicare fee-for-service CCM program itself, the care management tactics reported in this study may have broader applicability to enable high impact population health management in value-based care programs.

\section{Author Disclosure Statement}

The authors declared the following potential conflicts of interest: Dr. Mills, Mr. Douglas, Ms. Owens, Ms. Pridham, Ms. King, Ms. Roosa, Mr. Dznia, and Mr. Weber are employees of and stockholders in Chronic Care Management, Inc. None of these authors was involved with any aspect of the data analysis. All data and statistical analysis were performed by Dr. Poltavski, an independent statistician not affiliated with the company. Funding for this study was provided by Chronic Care Management, Inc.

\section{Supplementary Material}

Supplementary Table S1

\section{References}

1. Centers for Medicare \& Medicaid Services. Medicare Multiple Chronic Conditions 2015 data. https://www.cms .gov/Research-Statistics-Data-and-Systems/Statistics-Trendsand-Reports/Chronic-Conditions/MCC_Main.html. Accessed March 7, 2019.

2. Centers for Medicare and Medicaid Services. Chronic Care Management Toolkit. https:/www.cms.gov/About-CMS/ Agency-Information/OMH/Downloads/CCM-Toolkit-UpdatedCombined-508.pdf. Accessed March 7, 2019.

3. Shurrer J, O'Malley A, Wilson C, McCall N, Jain N. Evaluation of the diffusion and impact of the chronic care management (CCM) services: final report. Princeton, NJ: Mathematica Policy Research, 2017.

4. Yeager VA, Wharton MK, Monnette A, et al. Non-face-toface chronic care management: a qualitative study assessing the implementation of a new CMS reimbursement strategy. Popul Health Manag 2018;21:454-461.

5. O'Malley AS, Sarwar R, Keith R, Balke P, Ma S, McCall N. Provider experiences with chronic care management (CCM) services and fees: a qualitative research study. J Gen Intern Med 2017;32:1294-1300.

6. Basu S, Phillips RS, Bitton A, Song Z, Landon BE. Medicare chronic care management payments and financial returns to primary care practices: a modeling study. Ann Intern Med 2015;163:580-588.

7. Gardner RL, Youssef R, Morphis B, DaCunha A, Pelland $\mathrm{K}$, Cooper $\mathrm{E}$. Use of chronic care management codes for Medicare beneficiaries: a missed opportunity? J Gen Intern Med 2018;33:1892-1898.

8. Gagne JJ, Glynn RJ, Avorn J, Levin R, Schneeweiss S. A combined comorbidity score predicted mortality in elderly patients better than existing scores. J Clin Epidemiol 2011;64:749-759.
9. Flacker JM, Kiely DK. A practical approach to identifying mortality related factors in established long-term care residents. J Am Geriatr Soc 1998;46:1012-1015.

10. Centers for Medicare \& Medicaid Services. OASIS C-2 Guidance Manual. https://www.cms.gov/Medicare/QualityInitiatives-Patient-Assessment-Instruments/HomeHealth QualityInits/Downloads/OASIS-C2-Guidance-ManualEffective_1_1_18.pdf Accessed March 7, 2019.

11. Poe SS, Cvach MS, Dawson PB, et al. The Johns Hopkins fall risk assessment tool: postimplementation evaluation. J Nurse Care Qual 2007;22:293-298.

12. Kroenke K, Spitzer RL. The PHQ-9: a new depression and diagnostic severity measure. Psychiatric Ann 2002;32:509.

13. Kroenke K, Spitzer RL, Williams JB. The PHQ-9: validity of a brief depression severity measure. J Gen Intern Med 2001;16:606-613.

14. Berntsen GKR, Gammon D, Steinsbekk A, et al. How do we deal with multiple goals for care within an individual patient trajectory? A document content analysis of health service research papers on goals for care. BMJ Open 2015; 5:e009403.

15. Goldberg RM, Mabee J, Chan L, Wong S. Drug-drug and drug-disease interactions in the ED: analysis of a high-risk population. Am J Emerg Med 1996;14:447-450.

16. Health Leads. Social Determinants of Health Screening, Health Leads Toolkit. https://healthleadsusa.org/resourcelibrary/health-leads-tools/ Accessed March 7, 2019.

17. Bursac Z, Gauss CH, Williams DK, Hosmer DW. Purposeful selection of variables in logistic regression. Source Code Biol Med 2008;3:17.

18. Petrucci CJ. A primer for social worker researchers on how to conduct a multinomial logistic regression. J Social Serv Res 2009;35:193-203.

19. King EN, Ryan TP. A preliminary investigation of maximum likelihood logistic regression versus exact logistic regression. Am Stat 2002;56:163-170.

20. Tomz M, King G, Zeng L. Rare events logistic regression. J Stat Software 2003;8:1-27.

21. Steyerberg EW, Harrell FE Jr, Borsboom GJ, Eijkemans MJ, Vergouwe Y, Habbema JD. Internal validation of predictive models: efficiency of some procedures for logistic regression analysis. J Clin Epidemiol 2001;54:774781.

22. Lochner KA, Goodman RA, Posner S, Parekh A. Multiple chronic conditions among Medicare beneficiaries: statelevel variations in prevalence, utilization, and cost, 2011. Medicare Medicaid Res Rev 2013;3:pii: mmrr.003.03.b02.

23. Greysen SR, Stiljacic Cenzer I, Boscardin WJ, Covinsky KE. Functional impariment: an unmeasured marker of medical cost in older adults. J Am Geriatr Soc 2017;65: 1996-2002.

Address correspondence to: William R. Mills, MD

Chronic Care Management, Inc 30575 Bainbridge Road, Suite 300 Solon, $\mathrm{OH} 44139$

E-mail: william.mills@chroniccaremanagement.com 